\title{
Models of Identity Signaling
}

\author{
Paul E. Smaldino \\ Department of Cognitive \& Information Sciences \\ University of California, Merced \\ 5200 N. Lake Rd, Merced, CA 95343 USA \\ Email: psmaldino@ucmerced.edu
}

\begin{abstract}
Identity signals inform receivers of a signaler's membership in a subset of individuals, and in doing so shape cooperation, conflict, and social learning. Understanding the use and consequences of identity signaling is therefore critical for a complete science of collective human behavior. And, as with all complex social systems, this understanding is aided by the use of formal mathematical and computational models. Here I review some formal models of identity signaling. I divide these models into two categories. The first concerns models that assert how identity functions as a signal and test the consequences of those assertions, with a focus on public health behavior and disease transmission. The second concerns models used to understand how identity signals operate strategically in different social environments, with a focus on covert or encrypted communication.
\end{abstract}

Keywords: social identity, contagion, groups, modeling, agent-based models, cultural evolution

- - - In press at Current Directions in Psychological Science--—

Identities have behavioral and social consequences. In particular, a person's identity involves information that can be signaled (Smaldino, 2019a). Identity signals are those components of communication transmissions, displays, or other behaviors that inform receivers of the signaler's membership in some categorizable subset of individuals. These likely play a large role in human communication. Indeed, humans are nearly unique among vertebrates in our able to live in anonymous societies, because we can use identity signals rather than direct knowledge to identify others as targets for cooperation, social learning, avoidance, or aggression (Moffett, 2019). Certainly members of no other species live in societies of such complexity, enabled in part by our ability to make rapid decisions about how to interact with previously unknown individuals. In human societies, identity signals can be coarse-grained and overt, such as wearing clothing emblazoned with "Pride" rainbows or enthusiastically leading a "MAGA" rally, or they can take more covert forms like subtle fashion cues or political dog whistles. 
The intense sociality of human beings enables the benefits of large-scale cooperation, but also carries the risks of exploitation and conflict. Identity signals help to guide individual decisions regarding whom to associate with, learn from, avoid, or attack. As such, identity is entangled with many of our most urgent social problems (Bak-Coleman et al., 2021), including organized violence and oppression, misinformation, and the politicization of public health measures, sustainable practices, and climate change. Understanding how identity signals shape social behavior, and how the signals themselves are employed strategically, is therefore critical.

Knowing that identity signals are important is the first step, but actionable predictions and prescriptions requires the development of more precise theories of identity signaling. Ideally, theories about complex social systems-including those involving identity signaling-should be articulated sufficiently clearly that they can be formalized mathematically or computationally (Smaldino, 2017; 2020). Formal models help us to see the assumptions underlying our theories, and by establishing the consequences of those assumptions we can make broad predictions and identify important targets for future research. The program of producing formal models of identity signaling is still in a relatively early stage, but it is underway. In what follows, I present some formal models that explore the social consequences of identity signaling. I divide this presentation into two main sections. The first section concerns models that assert how identity is likely to function as a signal and test the consequences of those assertions on population-level dynamics. The second section concerns models that focus on understanding how identity signals are themselves shaped by population processes, and therefore likely to operate in different social environments.

\section{Asserting Identity: Outgroup Aversion and Disease Transmission}

In this first class of models, individuals are asserted to have observable identities that can be used to guide the decision making of others. In other words, features of psychology and behavior are taken as given, in order to understand their consequences at the population level. This connection is critical, because it is often difficult to predict how individual-level behaviors scale up to macro-level patterns, especially across time scales longer than those that can be studied experimentally (Resnick 1994). A classic example of this approach is Schelling's (1971) work on segregation, in which individuals use observable identities and weak preferences for similar neighbors to unconsciously assort into strongly segregated neighborhoods without ever intending to do so. Here I highlight work on identity signaling in a polarized population characterized by outgroup aversion - the tendency to avoid or disadopt products or behaviors that become associated with an outgroup-with special attention paid to the spread of infectious disease.

Many modeling frameworks for understanding social behavior have historically ignored identity, including models of infectious disease. There is a great opportunity to improve our understanding of public health and epidemiology by incorporating traditional modeling approaches with our understanding of how individuals use identity to assort with one another and adopt health-relevant behaviors (Moya et al., 2020). For example, consider vaccination rates and the phenomenon of herd immunity. Using standard epidemiological models, 
researchers can calculate the proportion of the population that would likely need to be vaccinated in order for the overall infection rate to steadily decline-to achieve "herd immunity." However, these models usually assume that vaccines are uniformly distributed in the population. Salathé and Bonhoeffer (2008) showed that if vaccinations tend to cluster in networks - that is, if people tend to assort into identity groups with others who share their views on vaccination - then the overall vaccination rate needed for herd immunity is likely to be substantially greater than what is predicted by the standard models. This indicates that incorporating identity into models of social behavior isn't merely interesting, it's greatly important.

If a population is polarized into opposed identity groups, then products or behaviors associated with the outgroup may become undesirable, even if they are intrinsically desirable in the absence of social comparisons (Berger \& Heath, 2007; 2008). When outgroup aversion between two groups is added to standard innovation diffusion models, it can lead to interesting and complex patterns of adoption (Smaldino et al. 2017; Smaldino \& Jones, 2021). Results from such a model are shown in Figure 1A, which plots the equilibrium adoption rate among each of two groups as a function of outgroup aversion. Adoption may decrease among both groups, with the group that adopts later experiencing a larger decline in adoption. If outgroup aversion is sufficiently strong, the behavior or adoption can become wholly associated with one group and not another, and thereby serve as a reliable social marker.
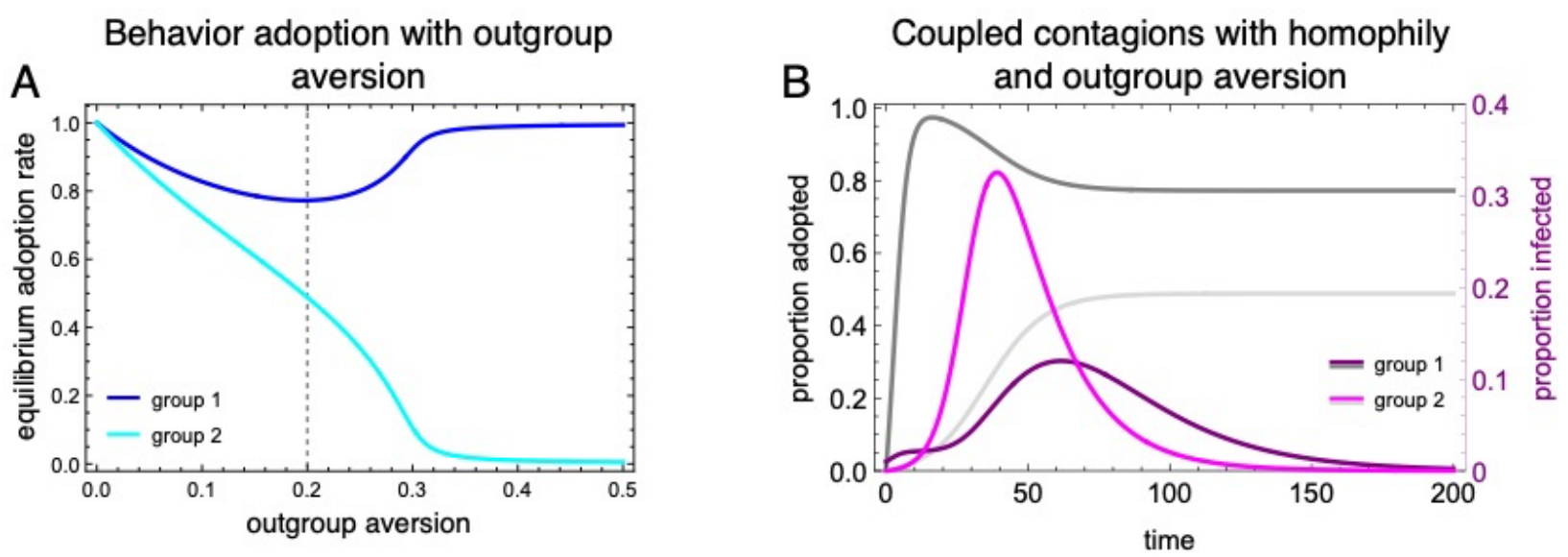

Figure 1. Outgroup aversion. (A) The equilibrium behavior adoption rates among two interacting groups as a function of outgroup aversion, where group 1 has a higher baseline adoption proclivity. (B) Coupled dynamics of preventive behavior adoption (shades of grey; group $1=$ dark, group $2=$ light) and disease infection (shades of magenta; group $1=$ dark, group 2 = light) among two interacting groups exhibiting both homophily (so that disease transmission occurs mostly within groups) and outgroup aversion (at a level indicated by the dashed vertical line in A). Adapted from Smaldino \& Jones (2021).

There has been increasing interest in coupled contagion models, in which both behavior and disease are modeled as contagions that influence one another (Funk et al., 2010; Verelst et al. 2016). Yet these models rarely incorporate variation in how particular individuals are likely to influence one another. James Holland Jones and I created a mathematical model in which the 
population was divided into two groups that interacted primarily with ingroup membersthey exhibited homophily (Smaldino \& Jones, 2021). A disease breaks out in one group, along with a behavioral contagion that decreases transmission among those who adopted it, capturing phenomena like social distancing and wearing face masks. Without outgroup aversion, the behavior spreads rapidly and the spread of the disease is suppressed. Things change when we include outgroup aversion. Beyond general support for this effect, research on the "behavioral immune system" suggests that outgroup aversion may be further strengthened when the threat of a harmful pathogen is salient (Schaller \& Park, 2011). When outgroup aversion is included in the model, the disease spreads much more widely. Although the disease first appears in group 1, infection ends up being much more rampant in group 2, whose members are more likely to avoid adopting the preventative behavior. Moreover, the increased disease prevalence in group 2 leads to a larger "second wave" in group 1 long after the initial outbreak (Figure 1B). While this model is fairly abstract, the results indicate that including behavioral nuances like identity clustering and outgroup aversion are important for making valid inferences about the dynamics of infectious disease and related social phenomena. Indeed, this model, first analyzed in the spring of 2020, anticipated some of the unfortunate dynamics related to COVID-19 transmission in polarized countries like the U.S., in which interventions like face masks and even vaccines became identity markers that were subsequently shunned by many who identified with oppositional groups, with tragic consequences (Johnson et al., 2020; Goodspeed, 2021).

\section{Understanding Identity: Covert and Overt Signaling}

Incorporating theories of identity enriches models of social dynamics and allows us to make more nuanced predictions about how those dynamics unfold. Models like the ones just presented tend to use fairly coarse-grained characterizations of identity, in which individuals belong to one of a discrete number of groups and are easily identified as members of those groups. This simplification is useful for providing a baseline for the effect of identity, and may be justified empirically in some cases. Nevertheless, identity signaling is often more nuanced. Individuals may conceal their identities entirely, or signal them covertly so that only those with insider knowledge are likely to perceive an identity signal for what it is. It is important to understand what this diversity in signaling behavior accomplishes, as well as the social forces that lead to reliable patterns in signaling behavior under varying constraints. This may subsequently allow us to better understand why we observe some of the variation in signaling behavior that we do. In this second class of models, we attempt to understand how strategies for identity signaling are shaped by social forces, and therefore which strategies are likely to be common in particular social environments.

\section{Identity Signaling Is For Cooperative Assortment}

To understand the subsequently presented models of identity signaling, I think it is useful to first take a brief detour to discuss the joint problems of cooperation and coordination, which identity signals appear to be partially designed to solve (Smaldino, 2019). Humans cooperate vastly, often with people who are unrelated to us by blood, and with people who we are likely to never see again. A great deal has been written on the free rider problem, which concerns 
how cooperative individuals can avoid being exploited by others (reviewed in Raihani, 2021). Although solving the free rider problem likely motivates much of human social behaviors and cultural institutions, another problem remains even when it is solved. Generating the benefits of cooperation involves finding the right people to cooperate with (Calcott, 2008; Smaldino, 2014). What makes a partner ideal for cooperation depends on one's own characteristics and the details of the cooperative task at hand. The problem then becomes one of assorting with sufficiently similar people so that cooperation is made efficient through coordination on norms, goals, and expectations. Even in cases where diversity or complementarity is preferable to perfect alignment, a baseline degree of similarity is still needed to facilitate coordination. For example, team performance often suffers when members have misaligned goals (Chow \& Chan 2008).

Here is where identity signaling comes into play. Norms, goals, and expectations-which I'll just call social traits - are often opaque. How are we to find those who share our social traits? If social traits correlate with group identities, the goal is straightforward: optimize coordination by assorting with ingroup members and avoid outgroup individuals. In theory, if a society is small enough that all ingroup members can be individually known, there is little need for signals to demarcate ingroup from outgroup individuals. In practice, the need to interact with outgroup individuals for things like trade and finding marriage partners nearly always requires a way to distinguish between strangers who are like us and strangers who are not. In contemporary societies we often consider complete strangers to be members of our ingroup if we perceive that they share the same nationality or religion, or support the same sports team, musical act, or political party.

In this "dividing line" scenario in which one assorts only with ingroup members, overt markers-which can be linguistic, behavioral, or sartorial—can serve to delineate potential cooperation partners from those to be avoided or even to be targets of aggression (Smaldino 2019a). Evolutionary models have shown how arbitrary markers can readily become associated with particular norms (McElreath et al. 2003). Of course, such a scenario is hardly limited to small-scale societies or ancient history; "us vs. them" distinctions are commonplace in today's world. However, the boundary between us and them is often more fuzzy than stark.

\section{A Theory of Covert Signaling}

The large, diverse societies in which most of us live are accompanied by special problems for identity signaling. It is rarely feasible to simply avoid or attack everyone who is not solidly a member our ingroup, not to mention the fact that the definition of ingroup is often contextspecific (Roccas \& Brewer, 2002; Smaldino, 2019a). We must work with dissimilar people, engage in commerce with them, and otherwise interact as functioning members of a diverse society. Nevertheless, some assortment is surely better than none, particularly if it allows cooperation toward goals that are not shared by everyone. Theories of identity signaling must therefore account for signaling strategies that are attuned to the complexity of contemporary, multicultural societies. This requires a radical rethinking of how identity is used for cooperative assortment, avoidance, and aggression in large, diverse societies (Smaldino, 2019a). To tackle one aspect of this problem, my colleagues and I have proposed and formalized a theory of covert signaling (Smaldino et al. 2018; Smaldino \& Turner, in press) that 
deals with how identity signaling works when overt "us vs. them" signaling is overly coarsegrained.

Covert identity signals are those aspects of communication that allow individuals who share social traits to recognize one another while simultaneously allowing signalers to avoid being recognized as dissimilar by those not "in the know" (Figure 2A). Political dog whistling is a well-discussed example. For example, former U.S. president George W. Bush often publicly denounced the 1857 Dred Scott Supreme Court decision. While opposition to an old, sinceoverturned judicial decision that upheld slavery may seem banal, the mention was interpreted by many evangelical conservatives as morally analogous to the 1973 Roe v. Wade case that upheld the right to abortion, and so communicated to these audiences the president's committed opposition to abortion rights (Kirkpatrick, 2004). More quotidian examples of covert signaling are well-documented, including the ways in which people implement fashion, humor, and other semiotic tools to subtly indicate identity (Smaldino \& Turner, in press). Covert signaling may be particularly important to members of persecuted minorities, such as LGBTQ+ individuals or political dissidents, who have strong incentives to assort but avoid detection by non-members. However, covert signaling is favored whenever the benefit of avoiding detection by dissimilar partners outweighs the cost of diminished signal strength.

The idea of covert signaling is not new; for example, Loury (1994) describes it quite explicitly in his discussion of political correctness. However, formal modeling allows us to more clearly articulate the theory and to ask more precise questions about the social conditions that might favor the use of covert signaling (Smaldino, 2017). The models include not only identity signaling strategies, but also strategies for processing that information, as well as a stage involving cooperative assortment in which agents use the information learned from identity signaling to form partnerships and receive associated payoffs (Smaldino et al. 2018; Smaldino \& Turner, in press). We vary the degree to which individuals are able to preferentially assort with partners they like and avoid partners they dislike (which we designated as homophily). Similar pairs get higher payoffs than dissimilar pairs, due to an enhanced ability to coordinate. If partners are dissimilar, they incur additional costs if they are aware of their dissimilarity, because their negative attitudes impair their ability to coordinate. The model assumes a (cultural) evolutionary dynamic, in which signaling strategies are copied from more successful individuals (Mesoudi, 2011; Kendal et al., 2018).

Analysis of the models reveals that covert signaling is favored when being disliked is costly and when individuals cannot count on being able to partner only with those they prefer (Figure 2B). This indicates that more covert signaling should be evident in scenarios consisting of arbitrary encounters that are likely to involve being paired with dissimilar partners. We explored this idea in greater depth, showing that covert signaling is increasingly favored in more diverse societies and among individuals with minority status (Figure 2C).

Treating identity signaling as a behavior with social consequences means that the models generate testable predictions about how people will actually use communication that carries identity information. Identifying covert signals is inherently difficult, however, because by definition they carry information accessible only to members of the associated identity group. 
My colleagues and I recently used differential responses by left and right partisans to tweets sent during a period of intense political discussion-the weeks before the 2020 U.S.

presidential election-to identify likely overt and covert signals (van der Does et al., 2021). We found that Twitter users with more heterogeneous follower networks tweeted more covertly. In a behavioral experiment in which participants were rewarded when selecting tweets that were liked and punished when selecting tweets that were disliked, we found that participants selected more covert signals when their audience consisted of more cross-partisans. These findings suggest the model captures something real about how people signal identity in a diverse society.

A
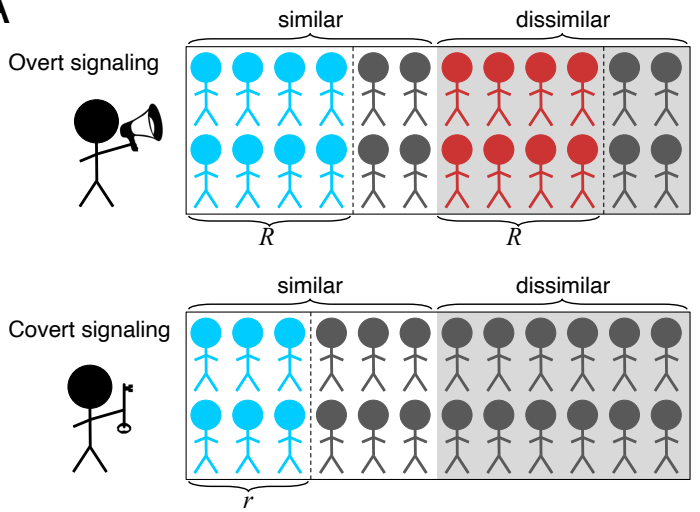

B

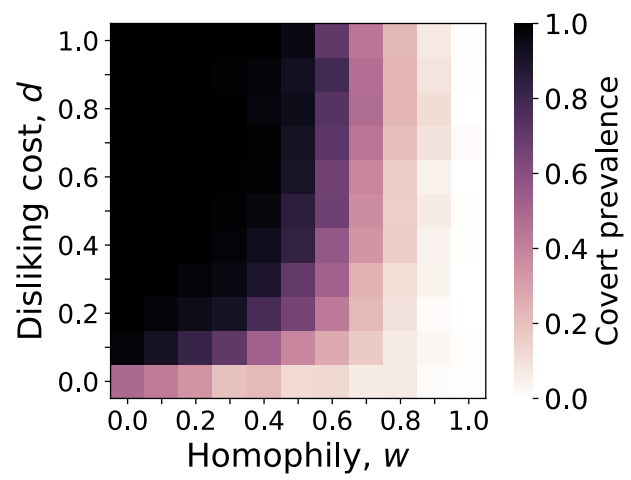

C
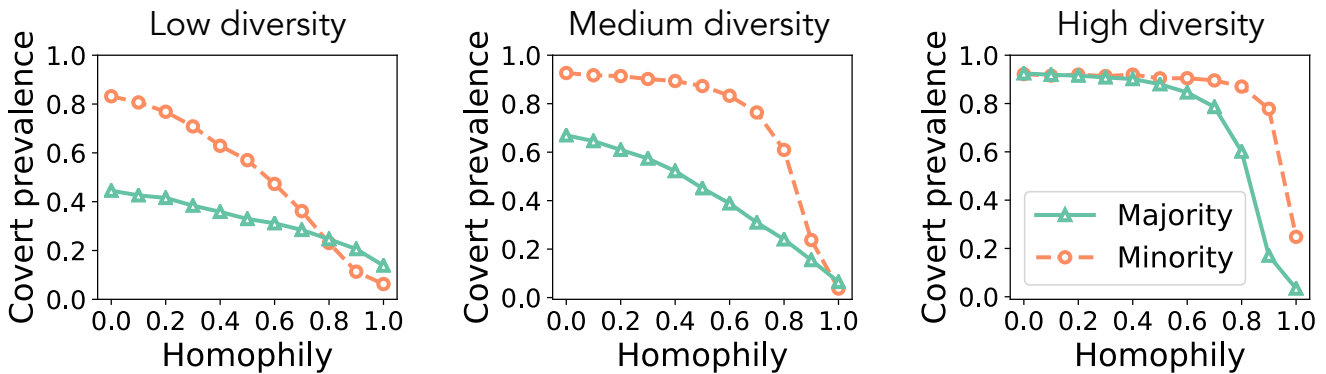

Figure 2. Covert signaling. (A) Identity signaling leads to liking by similar receivers and disliking by dissimilar receivers. Covert signaling is noisier but avoids detection by dissimilar receivers. (B) Equilibrium frequency of covert signaling of agent-based simulations as a function of homophily and the cost of being disliked. (C) Covert signaling increases in more diverse societies and among minority individuals. Adapted from Smaldino \& Turner (in press).

\section{Finding Out More About Who We Are}

A complete science of human social behavior requires a strong appreciation for the organizations and coalitions into which people sort themselves and each other, how the resulting identity designations influence social dynamics, and how the social environment shapes how individuals signal their identities and process identity information from others. Many questions remain in need of theories clear enough to be modeled. How do identities come to be represented in the mind? How might the size or diversity of a culture influence the 
nature of those identities, including tradeoffs between the depth vs. breadth of identity representations? How do subcultures emerge and evolve? To advance theory in these areas, additional modeling techniques may prove useful, such as the use of multilayer networks to represent the many social contexts in which humans interact (Atkisson et al., 2019). The ultimate goal is tight integration between models and empirical data (Smaldino, 2019b). This is difficult when most of our current measures of social identity are coarse grained, and rarely account for context. In the meantime, translating theories into formal models can help us to figure out what to look for.

\section{References}

Atkisson, C., Górski, P. J., Jackson, M. O., Hołyst, J. A., \& D'Souza, R. M. (2020). Why understanding multiplex social network structuring processes will help us better understand the evolution of human behavior. Evolutionary Anthropology, 29(3), 102-107.

Bak-Coleman, J. B., Alfano, M., Barfuss, W., Bergstrom, C. T., Centeno, M. A., Couzin, I. D., ... $\&$ Weber, E. U. (2021). Stewardship of global collective behavior. Proceedings of the National Academy of Sciences, 118(27).

Berger, J., \& Heath, C. (2008). Who drives divergence? Identity signaling, outgroup dissimilarity, and the abandonment of cultural tastes. Journal of Personality and Social Psychology, 95(3), 593.

Berger, J., \& Heath, C. (2007). Where consumers diverge from others: Identity signaling and product domains. Journal of Consumer Research, 34(2), 121-134.

Calcott, B. (2008). The other cooperation problem: Generating benefit. Biology \& Philosophy, 23(2), 179-203.

Chow, W. S., \& Chan, L. S. (2008). Social network, social trust and shared goals in organizational knowledge sharing. Information \& Management, 45(7), 458-465.

Funk, S., Salathé, M., \& Jansen, V. A. (2010). Modelling the influence of human behaviour on the spread of infectious diseases: a review. Journal of the Royal Society Interface, 7(50), 1247-1256.

Goodspeed, D. (2021). https://dangoodspeed.com/covid/total-cases-since-june

Johnson C. K., Fingerhut, H., \& Deshpande, P. (2020, November 5). Counties with worst virus surges overwhelmingly voted Trump. AP News. https://apnews.com/article/countiesworst-virus-surges-voted-trump-d671a483534024b5486715da6edb6ebf

Kendal, R. L., Boogert, N. J., Rendell, L., Laland, K. N., Webster, M., \& Jones, P. L. (2018). Social learning strategies: Bridge-building between fields. Trends in Cognitive Sciences, 22(7), 651-665.

Kirkpatrick, D. D. (2004, October 17). Speaking in the tongue of evangelicals. New York Times. https://www.nytimes.com/2004/10/17/weekinreview/speaking-in-the-tongue-ofevangelicals.html

Loury, G. C. (1994). Self-censorship in public discourse: A theory of "political correctness" and related phenomena. Rationality and Society, 6(4), 428-461.

McElreath, R., Boyd, R., \& Richerson, P. J. (2003). Shared norms and the evolution of ethnic markers. Current Anthropology, 44(1), 122-130.

Mesoudi, A. (2011). Cultural evolution. University of Chicago Press. 
Moffett, M. W. (2019). The human swarm: How our societies arise, thrive, and fall. Basic Books. Moya, C., Cruz y Celis, P., Kline, M. A., \& Smaldino, P. E. (2020). Dynamics of behavior change in the COVID world. American Journal of Human Biology, 32, e23485.

Raihani, N. (2021). The social instinct: How cooperation shaped the world. St. Martin's Press.

Resnick, M. (1994). Turtles, termites, and traffic jams: Explorations in massively parallel microworlds. MIT Press.

Roccas, S., \& Brewer, M. B. (2002). Social identity complexity. Personality and Social Psychology Review, 6(2), 88-106.

Salathé, M., \& Bonhoeffer, S. (2008). The effect of opinion clustering on disease outbreaks. Journal of The Royal Society Interface, 5(29), 1505-1508.

Schaller, M., \& Park, J. H. (2011). The behavioral immune system (and why it matters). Current Directions in Psychological Science, 20, 99-103.

Schelling, T. C. (1971). Dynamic models of segregation. Journal of Mathematical Sociology, 1, 143-186.

Smaldino, P. E. (2014). The cultural evolution of emergent group-level traits. Behavioral and Brain Sciences, 37(3), 243-295.

Smaldino, P. E. (2017). Models are stupid, and we need more of them. In R. Vallacher, S. J. Read, \& A. Nowak (Eds.), Computational social psychology (pp. 311-331). New York: Psychology Press. In R Vallacher, SJ Read, \& A Nowak (Eds.), Computational social psychology (pp. 311-331). New York: Psychology Press.

Smaldino, P. E., Janssen, M. A., Hillis, V., \& Bednar, J. (2017). Adoption as a social marker: Innovation diffusion with outgroup aversion. Journal of Mathematical Sociology, 41, 2645.

Smaldino, P. E., Flamson, T. J., \& McElreath, R. (2018). The evolution of covert signaling. Scientific Reports, 8, 4905.

Smaldino, P. E. (2019a). Social identity and cooperation in cultural evolution. Behavioural Processes, 161, 108-116.

Smaldino, P. (2019b). Better methods can't make up for mediocre theory. Nature, 575, 9.

Smaldino, P. E. (2020). How to translate a verbal theory into a formal model. Social Psychology, $51,207-218$.

Smaldino, P. E., \& Jones, J. H. (2021). Coupled dynamics of behaviour and disease contagion among antagonistic groups. Evolutionary Human Sciences, 3, E28.

Smaldino, P. E., \& Turner, M. A. (in press). Covert signaling is an adaptive communication strategy in diverse populations. Psychological Review.

van der Does, T., Galesic, M., Dunivin, Z., \& Smaldino, P. E. (2021). Strategic identity signaling in heterogeneous networks. PsyArXiv. https://psyarxiv.com/y5du7

Verelst, F., Willem, L., \& Beutels, P. (2016). Behavioural change models for infectious disease transmission: a systematic review (2010-2015). Journal of The Royal Society Interface, 13(125), 20160820. 


\section{Recommended Readings}

- Funk et al. (2010) (See References) A thorough review of coupled contagion models of behavior and disease transmission.

- Kauffman, S. A. (1971). Articulation of parts explanation in biology and the rational search for them. In R. C. Buck \& R. S. Cohen (Eds.), PSA 1970 (pp. 257-272). Philosophy of Science Association. An philosophical discussion of hypothesis generation and the value formal models in articulating and testing hypotheses.

- McElreath et al. (2003) (See References) This paper presents a simple model of social norms and ethnic markers, and introduces the relevant theory.

- Moffett, M. W. (2020). Societies, identity, and belonging. Proceedings of the American Philosophical Association, 164, 1-9. A brief summary of the research on human identity and anonymous societies; introducing many of the ideas fleshed out in Moffett (2019) in the References.

- Smaldino, P. E. (2019a) (See References). Reviews the role of identity signaling in cooperative assortment throughout human cultural evolution.

\section{Acknowledgments}

I thank Emily Newton and two anonymous reviewers for comments that improved this paper. The work presented here was funded by NSF RAPID grant BCS-2028160, ARO grant W911NF-20-1-0220, and by a Hellman Fellows Fund award. 